\title{
Research on Solution of Time Delay in Networked Control System
}

\author{
Fan Rong Wang ${ }^{1,2}$ \\ ${ }^{1}$ School of Electrical and Electronic Engineering, Hubei University of Technology,Wuhan, China \\ ${ }^{2}$ Department of Automation, Wuhan University, Wuhan,China \\ wfr@mail.hbut.edu.cn
}

Keywords: Networked control system; Time delay; Predictive control;

\begin{abstract}
There are problems such as time delay, packet loss in network systems. When it is introduced in traditional control systems as signal transmission medium,the control performance will be deduced.In this paper, a predictive control algorithm is used to study time delay in networked control system. Simulation results show that the network predictive control can ensure good control output.This paper focuses on mathematical realization of predictive control method for in detail and it plays an active role in guiding the engineering applications.
\end{abstract}

\section{Introduction}

With the rapid development of computer network communications and smart sensor technology, the computer control system transited from a centralized control to distributed control and field-bus control, network-based distributed control. This distributed work model thoroughly changed the traditional application of feedback control system, networked control systems (replication) arises at the historic moment. Document ${ }^{[1-5]}$ showed it generated directly from the development of the computer control technology and its wide application.

In 1962, Britain's imperial chemical industries pioneered computer direct digital control (DDC) system, adopting digital computer to replace the traditional analog controller as the core of system control cell;Honeywell in 1975 launched the first set of distributed control system (TDC-2000) as the computer network technology in control system of the early application results.Further into the 21 st century, industrial control technology with the combination of computer network technology has given birth to the field-bus system[6-9]. It has a stronger ability of digital computing and digital communication, which can realize the distributed control system.

Compared with the traditional point-to-point control systems, network control system has a lot of advantages such as lower system connection, flexible structure, opening communication protocol. But communication network in the traditional control system makes the network control system analysis and design become more complex, unreliable network transmission mode leads to a lot of uncertainty, the main problems are: the network induced delay, data packet loss, packet sequence disorder, noise and so on.

In order to solve the data transmission multiple cycle delay problems[10-13] in network control system, this paper uses predictive control algorithm, multistep test, rolling optimization and feedback correction control strategy to improve the system, And the control effect under congestion environment is improved by optimizing the control sequence.

\section{Network control circuit design}

Network predictive control structure. In the network control system, the main characteristics of the system configuration is that the sensor, the controller and the actuator are not directly connected by point-to-point,using the public network,the data and control information exchange ${ }^{[14-15]}$ through a serial digital communication interface. All devices share a signal transmission channel to realize the data communication between devices, resource sharing and mutual cooperation. Typical network control system structure is shown as Fig.1. In the NCS, 
there are two categories of the nodes drive way: the clock driver class and event-driven class. Under normal circumstances, the NCS sensor uses clock drivers.In this paper, network predictive control is divided into two parts:the controller side and the controlled object side, and the whole structure of the control loop is shown as Fig.2. When the controller receives the data from feedback channel, the event is triggered, then the controller will generate the corresponding predicted sequence.Using the system clock as a drive mode, the controlled object side takes control sequences as data source and selects corresponding control signals to control the specific device. These control sequences can be achieved on the network delay compensation.

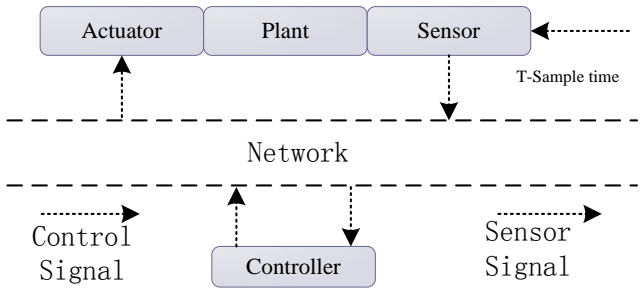

Figure. 1 NCS Structure

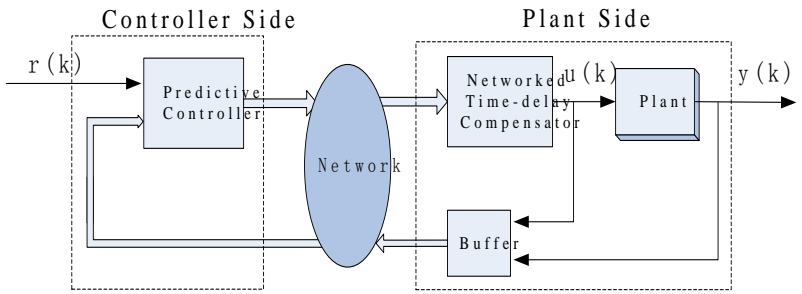

Figure. 2 Network Predictive Control Structure

Equipment model and local control. Assuming that the system input is $u(t)$ and the output is $y(t)$ at time $t$, the input and out put will satisfy a basic linear differential equation.

$$
y(t)+a_{1} y(t-1)+\cdots a_{n} y(t-n)=b_{1} u(t-1)+\cdots b_{m} u(t-m)
$$

Using the above representation, associated observational data of the discrete-time system model can be directly related because the data are often obtained by sampling. In Equation 1, for convenience of description, assuming that the sampling time interval is a unit of time. With simple transformation, it becomes a formula which can obtain the output according to the observation results given previously.

$$
y(t)=-a_{1} y(t-1)-\cdots-a_{n} y(t-n)+b_{1} u(t-1)+\cdots+b_{m} u(t-m)
$$

This paper takes the basic SISO local control system as an example and describe its mathematical model. The most basic local control system structure is shown in Figure 3. The reference input signal is $r$. The controller output signal is $u(t)$. The device output signal is $y(t)$.

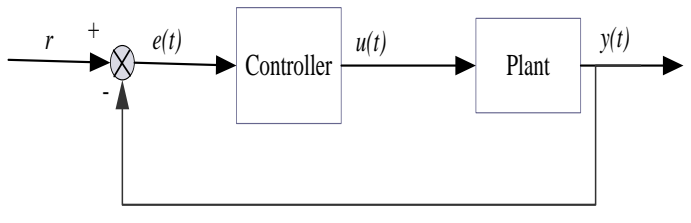

Figure. 3 SISO local control system

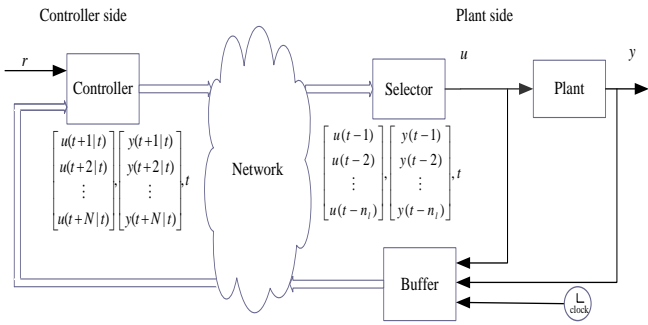

Figure. 4 Network prediction control data flow graph

In the network control system, since all the signals are digitized and the data are obtained by sampling, the device adopts discrete mathematical model. The device input and output satisfy the following relationship.

$$
y(t)=-a_{1} y(t-1)-\cdots-a_{n_{a}} y\left(t-n_{a}\right)+b_{1} u(t-1)+\cdots+b_{n_{b}} u\left(t-n_{b}\right)
$$

Because it is a constant state system, the device specific mathematical model can be obtained by system identification., and the value of constant coefficients ai and bi (i=1,2, $\cdots n$ ) can be obtained through the associated transform computation. The controller input $\mathrm{e}(\mathrm{t})$ and output $\mathrm{u}$ (t) satisfy the following relationship.

$$
u(t)=-c_{1} u(t-1)-\cdots-c_{n_{c}} u\left(t-n_{c}\right)+d_{0} e(t)+d_{1} e(t-1)+\cdots+d_{n_{d}} e\left(t-n_{d}\right)
$$

Predictive controller design. Network nodes are introduced to the local control system, and data flow diagram with a corresponding control sequence is shown in Fig.4. The control side is mainly composed of predictive controller predictive controller is responsible for receiving 
corresponding control sequence from feedback channel which is generated by recursive formula.The device side undertakes control sequence data through the selector, and selects control data as a device input. When the device side finished, it packages the corresponding data and transmits them back to the other side of the network controller via a feedback channel.

The prediction model has a function of expressing the future behavior of the system. It can calculate the output predicted trajectory, so it is possible to select the optimal control strategy to optimize a certain performance indicators.

The device side is driven by clock. In the beginning of each sampling period, the occurred control sequence $\mathrm{u}(\mathrm{t})$, the output sequence $\mathrm{y}(\mathrm{t})$ and time point $\mathrm{t}$ are stored in a data packet, and then they are transmitted through the feedback channel to controller side.

The controller side is driven by event. When the packet is received from the side of the device through feedback channel, the controller will calculate predicted sequence in the future.by the recursive formula of Equation 2 and 3, and then send the predicted sequence together with the system time $\mathrm{t}$ to the device side.

Device side data selector. Most communication networks such as TCP/IP, data is transmitted in the form of the package.Networked control system can transfer a set of predicted sequent signal from network data senders through the same package, then the receiver selects an appropriate value of the signal according to the network conditions at the time. So it can improve the delay, packet loss and other unfavorable factors caused by the network transmission. There are several kinds of receiving packets anomalies packet delay,packages accumulation, packet loss. To solve these problems, this article assumes that the Network transmission delay has an upper limit $\mathrm{m}$ and time interval of continuous packet loss is not greater than the maximum delay $\mathrm{m}$. So these problems can be converted into a kind of random delay problems. Fig. 5 shows the principle.

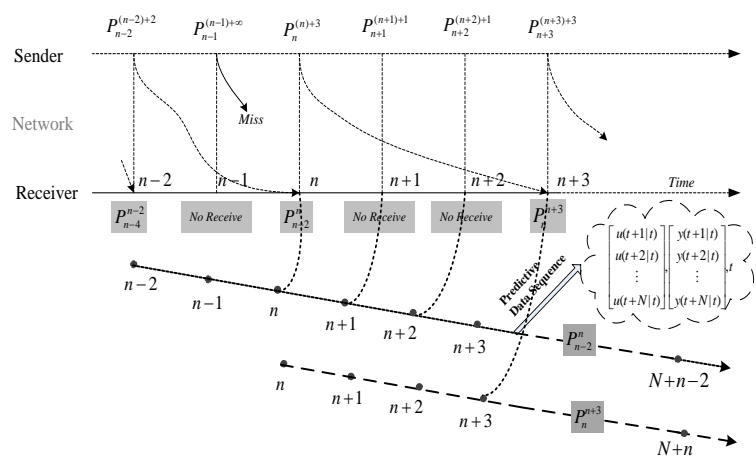

Figure. 5 Random Delay network solutions

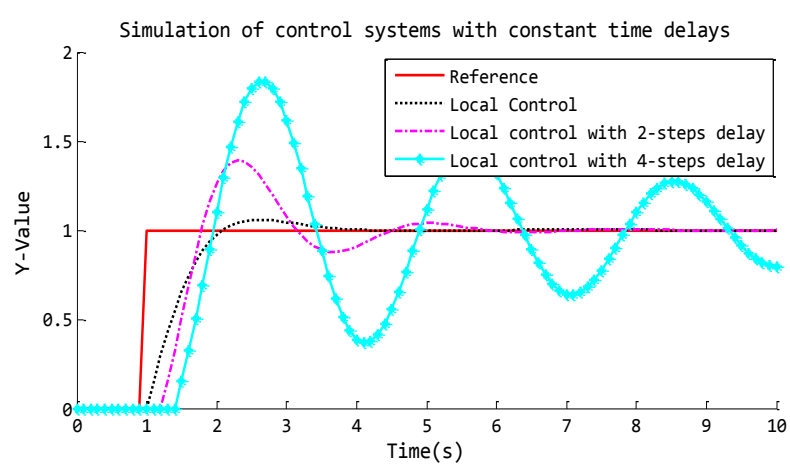

Figure. 6 Local fixed delay control system output

In the receiving end,the packet delay occurs at time node $n$, it received data packet of time node $\mathrm{n}-2$ from the transmission end, this packet has a delay of two sampling cycles. The conventional control method clearly does not meet the control requirements. But if the predicted sequence is used, the device-side data selector can choose the control value of time node $\mathrm{n}$ from the predicted sequence according to the time delay.And it can effectively compensate for time delay.

\section{Algorithm Simulation}

Mathematical model of the devices and controllers. In local control of the system depicted in Figure. 3, we set sampling cycle is $0.1 \mathrm{~s}$, and then digitize conventions related links. 
$\mathrm{r}(\mathrm{t})$ unit step input:

$$
1(t)=\left\{\begin{array}{l}
1, t \geq 0 \\
0, t<0
\end{array}\right.
$$

Device model and PI controller:

$G\left(z^{-1}\right)=\frac{B\left(z^{-1}\right)}{A\left(z^{-1}\right)}=\frac{0.4662 z^{-1}-0.2843 z^{-2}}{1-1.396 z^{-1}+0.4681 z^{-2}} \quad G\left(z^{-1}\right)=\frac{D\left(z^{-1}\right)}{C\left(z^{-1}\right)}=\frac{0.33-0.23 z^{-1}}{1-z^{-1}}$

In Fig. 6,it is easy to see, that local control system without any delay is able to achieve a good result, the output value of the equipment can quickly reach the reference input value. When 2 or 4 sampling period delay added to the local control system, the system output curve is oscillating, and the higher the fixed delay, then the oscillation of the system is more severe.

Network Predictive Control. The network system is a complex system, in addition to the fixed delay, it will produce more random delay. In the simulation design with random delay network environment, the paper sets that the network random delay range is limited to a fixed range.

In the prior agreement network the minimum and maximum delay, respectively, is 2 to 8 sampling period ,i.e.0.2s and $0.8 \mathrm{~s}$, a random delay link to a clock signal as an input, the output result is shown in Fig.7.

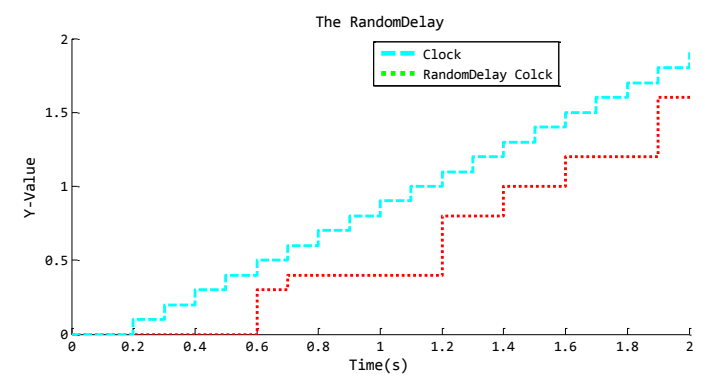

Figure. 7 Random delay loop output renderings

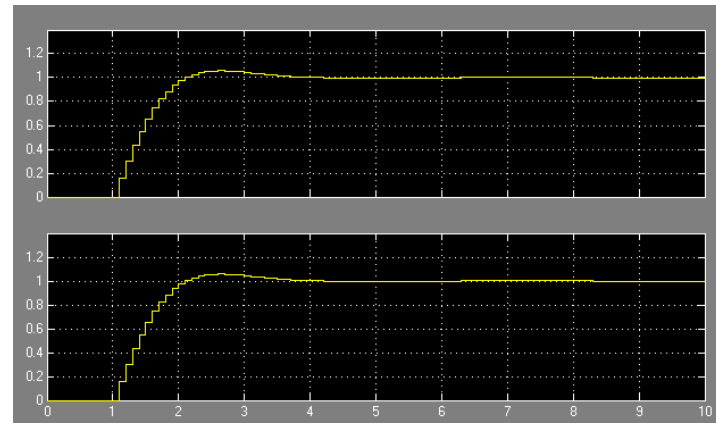

Figure.8 Local control (top) and NPC (bottom) device output

Fixed delay and random delay added to local control network latency environment, we get a new network control system. We change he device model and PI controller model to differential structure:

$$
\begin{aligned}
& y(t)=1.396 y\left(t-T_{s}\right)-0.4681 y\left(t-2 T_{s}\right)+0.4662 u\left(t-T_{s}\right)-0.2843 u\left(t-2 T_{s}\right) \\
& u(t)=u\left(t-T_{s}\right)+0.33 e(t)-0.23 e\left(t-T_{s}\right)
\end{aligned}
$$

It can be seen that the differential 4 and 5 already have speculated that the future sequence by sequence. Because the order of mathematical model of the system is no more than 2, generally, the value of $y$ and $u$ of current time can be obtained through the last two sets of $y$ and $u$. The two sets of differential recursive algorithm is the core of predictive controller. Occurred $\mathrm{u}, \mathrm{y}$ sequence and the current time value are transformed from the feedback channel after a certain network delay. Predictive controller uses them as input to get the future value and then transmits value through the network to the controller side of the data selector.

Data selector receives data packet from the controller side and selects the control value from the $u$ sequence. It may achieve effective compensation to network delay problem according to the the NPC solution. Fig.8 shows the local control (top) and network predictive control (bottom) device output map. It is easy to see that in the network control system, as long as the device and the controller model is sufficiently accurate, the control of the network system with the network predictive controller compensation can reach exactly the same effect as local control without delay. 


\section{Summary}

This paper introduces the basic principle of network time delay predictive control and implementation of the concrete mathematics method. And it takes the SISO network control system with a random time delay as an example. Results show that the NPC algorithm can realize effective compensation of network delay, so even in the presence of network delay uncertain environment, the controller is able to achieve good control effect. In this paper, the specific implementation steps of network control technology is described in layman's language from the engineering application level. It has played an active role in the promotion of he practical application of network predictive control technology.

\section{References}

[1]Halevi Y, Ray A. Integrated communication and control systems: Part I-Analysis[J]. Journal of Dynamic Systems, Measurement and Control. 1988, 110(4): 367-373.

[2]Nilsson J. Real-time control systems with delays[D]. Ph. D. dissertation, Department of Automatic Control, Lund Institute of Technology, 1998.

[3]Walsh G C, Ye H, Bushnell L G. Stability analysis of networked control systems[J]. Control Systems Technology, IEEE Transactions on. 2002, 10(3): 438-446.

[4]Lian F L, Moyne J R, Tilbury D M. Performance evaluation of control networks: Ethernet, ControlNet, and DeviceNet[J]. Control Systems, IEEE. 2001, 21(1): 66-83.

[5]Pleinevaux P, Decotignie J D. Time critical communication networks: Field buses[J]. Network, IEEE. 1988, 2(3): 55-63.

[6]Schickhuber G, Mccarthy O. Distributed fieldbus and control network systems[J]. Computing \& Control Engineering Journal. 1997, 8(1): 21-32.

[7]Hu W S, Liu G P, Rees D. Networked predictive control over the Internet using round-trip delay measurement[J]. Instrumentation and Measurement, IEEE Transactions on. 2008, 57(10): 2231-2241.

[8]Liu G P, Xia Y, Rees D, et al. Design and stability criteria of networked predictive control systems with random network delay in the feedback channel[J]. Systems, Man, and Cybernetics, Part C: Applications and Reviews, IEEE Transactions on. 2007, 37(2): 173-184.

[9]Liu G P, Xia Y, Chen J, et al. Networked predictive control of systems with random network delays in both forward and feedback channels[J]. Industrial Electronics, IEEE Transactions on. 2007, 54(3): 1282-1297.

[10]Hu W S, Liu G P, Rees D. Event-driven networked predictive control[J]. Industrial Electronics, IEEE Transactions on. 2007, 54(3): 1603-1613.

[11]Walsh G C, Beldiman O, Bushnell L G. Error encoding algorithms for networked control systems[J]. Automatica. 2002, 38(2): 261-267.

[12]Liu G P, Mu J X, Rees D, et al. Design and stability analysis of networked control systems with random communication time delay using the modified MPC[J]. International Journal of Control. 2006, 79(4): 288-297.

[13]Liu G P, Rees D, Chai S C. Design and practical implementation of networked predictive control systems[C]. IEEE Networking, Sensing and Control, ICNSC2005 - Proceedings. 2005. 336-341. 
[14]Zhao Y B, Liu G P, Rees D. Design of a packet-based control framework for networked control systems[J]. Control Systems Technology, IEEE Transactions on. 2009, 17(4): 859-865.

[15]Liu G P. Design, simulation and implementation of networked predictive control systems[J]. Measurement and control. 2005, 38(2): 17-21. 